\title{
Transcriptional analysis of in vitro expression patterns of Chlamydophila abortus polymorphic outer membrane proteins during the chlamydial developmental cycle
}

\author{
Nicholas Wheelhouse*, Kevin Aitchison, Lucy Spalding, Morag Livingstone, \\ David LONGBOTTOM
}

\author{
Moredun Research Institute, Pentlands Science Park, Bush Loan, Edinburgh, \\ Midlothian, EH26 0PZ, United Kingdom
}

(Received 17 March 2009; accepted 19 May 2009)

\begin{abstract}
Chlamydophila abortus is the aetiological agent of ovine enzootic abortion. Sequencing, annotation and comparative analysis of the genome of C. abortus strain S26/3 has revealed variation in the loci encoding the polymorphic membrane proteins (Pmps). These Pmps resemble autotransporter proteins of the type V secretion system, suggesting an important role in chlamydial pathogenesis. The purpose of this study was to characterise the transcriptional expression patterns of this family during the developmental cycle of $C$. abortus. McCoy cells were infected with C. abortus and analysed for pmp mRNA expression over a $72 \mathrm{~h}$ period. Few pmp transcripts were detected in the early stages of the developmental cycle. Peak expression occurred at $48 \mathrm{~h}$ post-infection (p.i.) other than for pmp5E, where it was observed at $24 \mathrm{~h}$ p.i. Overall, expression of pmps $5 E, 18 D$ and $10 G$ were found to be 40 to 100 -fold higher than the lowest expressing pmps $(6 \mathrm{H}, 13 \mathrm{G}$ and $15 \mathrm{G})$ at $24 \mathrm{~h}$ p.i., while pmps $18 \mathrm{D}$ and $17 G$ were 14 to 16 -fold higher than the lowest $(11 G, 14 G$ and $15 G)$ at $48 \mathrm{~h}$. Levels of expression for all the other pmp genes were below one copy per genome at any time point. The expression of all the pmps reduced to near base-line levels by $60 \mathrm{~h}$ p.i. These results demonstrate that pmp expression in $C$. abortus is mid to late cycle, consistent with conversion of the reticulate body to the elementary body. The low level of $p m p$ transcription may be indicative of heterogeneity in expression, suggesting a possible role for some of the Pmps in antigenic variation and chlamydial pathogenesis.
\end{abstract}

Chlamydophila abortus / pmp / gene expression / antigenic variation

\section{INTRODUCTION}

Chlamydophila abortus is the aetiological agent of ovine enzootic abortion (OEA), the single most common infectious cause of ovine abortion in the United Kindgom [1]. It is also a major cause of lamb mortality throughout Europe and is endemic in most sheep-rearing areas of the world. In addition $C$. abortus is

\footnotetext{
* Corresponding author: nick.wheelhouse@moredun. ac.uk
}

zoonotic and poses a potential risk to the health of pregnant women [16]. In common with other members of the Chlamydiaecae, C. abortus is a Gram-negative obligate intracellular pathogen that undergoes a biphasic life-cycle. The infectious form of the organism, the elementary body (EB), enters the host cell where it resides within a vacuole known as an inclusion. Within this inclusion the EB undergoes conversion to the metabolically active reticulate body $(\mathrm{RB})$, which replicates through binary fission. Between 48 and $72 \mathrm{~h}$ following infection the 
$\mathrm{RB}$ re-condense to $\mathrm{EB}$ and at the end of the cycle the inclusion and host cell are lysed releasing infectious organisms that infect neighbouring cells [16].

Sequencing, annotation and comparative analysis of the genome of $C$. abortus strain S26/3 has revealed variation in the loci encoding a family of outer membrane proteins known as polymorphic membrane proteins or Pmps [31], which were originally identified through their immuno-reactivity with convalescent sheep sera obtained from aborted ewes $[14,15]$. The expression of Pmps is not restricted to C. abortus and ртр genes have been identified in all pathogenic chlamydial species sequenced to date $[2,23,24$, 27, 31]. Whilst all sequenced Chlamydiaceae species possess pmp genes, there is widespread heterogeneity in the array of the genes carried by each species. While $C$. trachomatis and the closely related $C$. muridarum genomes encode 9 pmp genes (termed A-I), C. pneumoniae, C. abortus, C. caviae and C. felis genomes encode 21, 18, 17 and 20 pmps, respectively. These Pmps resemble autotransporter proteins (AT) of the type V secretion system in which the gene encodes a single precursor containing three functional domains, an N-terminal signal sequence, a passenger (effector) and a carboxyterminal $\beta$-barrel translocator domain [11]. In silico analyses of the Pmps have identified homology with several proteobacterial virulence factors secreted via the TypeV secretion apparatus, including the Escherichia coli adhesin AIDA-I [10]. Indeed a role in bacterial adhesion has been ascribed to PmpD [33] and yet whilst little else is understood about the functions of the Pmps it has also been suggested through genetic comparisons of $C$. trachomatis strains that they may play important roles in chlamydial pathogenesis [28] and niche specificity [7, 21]. Immunologically phase-variation in the expression of the Pmps through slip-strand slippage may play a role in the evasion of host immune responses $[22,31]$. In terms of their importance to the host immune response to chlamydial infection, Pmps induce both antigen-specific $\mathrm{T}$ cell responses $[8,26]$, essential in the clearance of primary infection [5] and specific humoral responses $[12,15]$ making them potential vaccine [17] and diagnostic candidates [25].
Little is known about the regulation or function of the Pmps in C. abortus and in Chlamydia generally. Given the potential importance of the Pmps in the virulence and pathogenesis of $C$. abortus and their potential in the development of vaccine and diagnostic reagents, the purpose of this study was to further characterise this important protein family by studying their expression during the developmental cycle through transcriptomic analysis.

\section{MATERIALS AND METHODS}

\subsection{Cell culture and $C$. abortus propagation}

McCoy cells were grown in RPMI1640 medium supplemented with $5 \%$ heat inactivated fetal calf serum. HEp2 cells used for the propagation and titration of $C$. abortus stocks were routinely grown in Iscove's Modified Dulbecco's Medium (Invitrogen, Paisley, UK) supplemented with $10 \%$ heat inactivated fetal calf serum (PAA Laboratories Ltd, Yeovil, Somerset, UK).

\subsection{Infection of cells and nucleic acid extraction}

The C. abortus strain S26/3 was grown in HEp2 cells and titrated according to a previously published protocol [9]. To investigate the in vitro expression of pmps in C. abortus, McCoy cells were grown to subconfluence in T25 tissue culture flasks (Corning Costar, High Wycombe, UK). Duplicate flasks were infected with $C$. abortus at an estimated multiplicity of infection (MOI) of two in infection medium consisting of RPMI 1640 containing 2\% FCS and $1 \mu \mathrm{g} / \mathrm{mL}$ cycloheximide (Sigma Aldrich, Dorset, UK). After $2 \mathrm{~h}$, the medium was removed and replaced with fresh infection medium. At $6,12,24$, $36,48,60$ and $72 \mathrm{~h}$ post-infection (p.i.) total DNA and RNA were isolated from paired flasks using DNeasy ${ }^{\circledR}$ and RNeasy ${ }^{+\circledR}$ mini kits (Qiagen, Crawley, West Sussex, UK). Experiments were conducted on three separate occasions.

\subsection{Nucleic acid quantitation and reverse- transcription (RT)}

DNA and RNA concentrations were quantified at $260 \mathrm{~nm}$ using a nanodrop ND-1-1000 spectrophotometer (ThermoFisher Scientific, Loughborough, Leicestershire, UK) and the quality of total RNA 
was assessed on an Agilent 2100 Bioanalyzer (Agilent, West Lothian, UK) prior to reverse transcription using an Omniscript ${ }^{\circledR}$ RT Kit (Qiagen) and random primers (Invitrogen).

\subsection{Generation of plasmids for standard curves}

Unique regions of each of the $p m p$ genes were amplified by PCR using specific primers (Tab. I) and an Expand Long-Template PCR kit (Roche Diagnostics, Burgess Hill, UK). PCR products were purified using the QIAquick ${ }^{\circledR}$ gel extraction kit (Qiagen) prior to cloning into the appropriate vector. Purified $16 \mathrm{~S}, 5 \mathrm{E}$ and ompl PCR products were cloned into $\mathrm{pGEM}^{\circledR}-\mathrm{T}$ Easy (Promega, Southampton, Hampshire, UK) the remaining pmp products had been previously cloned into the pET30-Ek/Lic vector (Merck chemicals Ltd. Nottingham, UK). Plasmids were either transformed into NovaBlue (Merck chemicals Ltd; pET30-Ek/Lic constructs) or JM109 (Promega; pGEM ${ }^{\circledR}$-T Easy constructs) competent $E$. coli cells according to standard procedures, and transformants were selected on the basis of appropriate antibiotic resistance. Colonies were selected for overnight culture and plasmids were purified using the QIAprep ${ }^{\circledR}$ spin miniprep kit (Qiagen). The presence of correct sequences was verified by sequencing on a MegaBACE ${ }^{\mathrm{TM}}-500$ capillary DNA sequencer using DYEnamic ${ }^{\mathrm{TM}}$ ET Dye Terminator technology (GE Healthcare, Little Chalfont, Bucks, UK).

\subsection{Real-time polymerase chain reaction (qPCR)}

Unique primers and probes were designed for each of 15 pmps and $16 \mathrm{~S}$ rRNA genes (Tab. II) using Primer Express v2.0 (Applied Biosystems, Warrington, Lancashire, UK). Real-time PCR protocols for quantitation of both omp1 [13] and C. abortus genomes [4] have been previously described. Assays were set up using TaqMan ${ }^{\circledR}$ Universal PCR Master Mix and run on an ABI PRISM 7000 Sequence Detection System (Applied Biosystems) according to standard procedures. In all RNA determinations, both forward and reverse primers were used at $900 \mathrm{nM}$ and probe at $250 \mathrm{nM}$ final concentration. Samples were analyzed in triplicate either with $1 \mu \mathrm{L}$ eluted DNA (for genome determination) or 5 ng RNA from each time point. For quantitation, relevant standard curves were also included on each plate. The number of mean copies per well were calculated against standard curves $\left(10^{8}-10^{2}\right)$ derived either from purified genomic DNA (to determine number of $C$. abortus genome copies) as previously described [13] or from plasmid (to determine transcript levels) for each relevant assay. Total numbers of recoverable genomes and transcripts were calculated per flask and transcripts were normalised against genomes for each time point from the duplicate flasks.

\subsection{Statistics}

Data were analysed by ANOVA (Genstat version 7 statistical package) using Fisher's least significant difference test to separate the means at both the $5 \%$ and $1 \%$ probability levels.

\section{RESULTS}

\subsection{RNA profiles}

Total RNA was prepared from C. abortus infected McCoy cells at 6, 12, 24, 36, 48, 60 and $72 \mathrm{~h}$ p.i. Figure 1 shows typical RNA profiles for two of the time points, 24 and $48 \mathrm{~h}$ p.i. Between 0 and $36 \mathrm{~h}$ there was little observed prokaryotic ribosomal RNA visible, however the eukaryotic RNA was intact. At $48 \mathrm{~h}$ there was a substantial increase in the levels of prokaryotic RNA observed and this coincided with a reduction in the levels of host RNA which was visible as a decrease in the overall intensity of the signals produced on the electropherogram. Post $48 \mathrm{~h}$ (60 and $72 \mathrm{~h}$ ) during which time there was significant levels of cell lysis there was an abrupt decrease in the levels of both eukaryotic and prokaryotic RNA.

\subsection{Expression of $16 \mathrm{~S}$ rRNA and ompl transcripts}

As an initial step in the analysis of the pmps, the expression of $16 \mathrm{~S}$ rRNA and ompl, both of which are considered to be constitutively expressed throughout the chlamydial developmental cycle, was determined and normalized relative to that of chlamydial genome number (Fig. 2). At the earliest time-points of 6 and $12 \mathrm{~h}$ p.i. there was negligible expression of the16S rRNA gene (Fig. 2A). There was a significant rise above the base-line levels of $16 \mathrm{~S}$ between 12 and $24 \mathrm{~h}$ p.i. $(p<0.05)$, which continued to increase at $36 \mathrm{~h}(p<0.01$ above 
Table I. Primers used to clone $p m p$ plasmid standards used in RT-PCR.

\begin{tabular}{|c|c|c|c|}
\hline Gene & Forward primer $\left(5^{\prime}-3^{\prime}\right)^{*}$ & Reverse primer $\left(5^{\prime}-3^{\prime}\right)^{*}$ & $\begin{array}{l}\text { Amplicon } \\
\text { size (bp) }\end{array}$ \\
\hline pmplB & GACGACGACAAGATCTCGATTACAGTATTTGGAGAAC & GAGGAGAAGCCCGGTTTAGAATATCATACGTGCGCCGCAGTTAG & 5351 \\
\hline pmp $2 A$ & GACGACGACAAGATTTCACTCGCACCACTGACTC & GAGGAGAAGCCCGGTTTAGAAACTTAGAGATATACCACC & 2792 \\
\hline pmp $3 E$ & GACGACGACAAGATTTTTACCTCTATTCTCGGAAGCC & GAGGAGAAGCCCGGTCTAAAAAATTAACGAGCTCCCTGC & 2937 \\
\hline pmp $4 E$ & GACGACGACAAGATCGAACTCATTTGCCTTGCTAAG & GAGGAGAAGCCCGGTCTAAAATTGTAATTCACTTG & 2807 \\
\hline pmp $5 E$ & ATAAGACCTGAGAAAACGATATGAAATCCAC & AGTTGCTAGAGAGTTTAGAAGGAAAGTTTGCCCCCAG & 1114 \\
\hline ртрб $6 H$ & GACGACGACAAGATTTGCTTATGCAGCTCGGGGTGG & GAGGAGAAGCCCGGTTTAGAATGTGCCTTGCAAGCCTGTGATG & 2930 \\
\hline $\operatorname{pmp} 7 G$ & GACGACGACAAGATTAACGGCGACCTTAGAGAGATGTTTC & GAGGAGAAGCCCGGTTTAGAATCGAAATTTACTTCCTAC & 3002 \\
\hline pmp10G & GACGACGACAAGATTGACGGCTCTACAGGGACC & GAGGAGAAGCCCGGTTTAACGAAGTTCGCCATTGCCTGA & 2408 \\
\hline pmp $11 G$ & GACGACGACAAGATTCTGGCAACTGGATTCAACGGC & GAGGAGAAGCCCCGGTTTAAAAATGCACCCTGCTG & 2495 \\
\hline pmp $13 G$ & GACGACGACAAGATCACGAACAGTGAGCCATTCAATC & GAGGAGAAGCCCGGTTTAGAAGCCAAATTGACTGAAGAGC & 2399 \\
\hline pmp $14 G$ & GACGACGACAAGATCGACGCAAACGGAGCATTCAG & GAGGAGAAGCCCGGTTTAGAATGCGACCTTAGCG & 2699 \\
\hline pmp $15 G$ & GACGACGACAAGATAAGCTCTAGCGATAATTACGATGG & GAGGAGAAGCCCGGTTTAGAATTTGTATCTACCC & 4110 \\
\hline pmp16G & GACGACGACAAGATCTCCTATGCAGGCAAAGATTCTCC & GAGGAGAAGCCCGGTTTACAAGCCAAATTGACTGAAGAGC & 2321 \\
\hline pmp $17 G$ & GACGACGACAAGATCTCCTTTGCAGGGAAAGATTCAGG & GAGGAGAAGCCCGGTTTAGGTTCGTGAAGATCCCCTGAGC & 2324 \\
\hline pmp $18 D$ & GACGACGACAAGATGTCTACATTTTCTCACTCCG & GAGGAGAAGCCCGGTTTAGAAGATCAAGCGCATACCACAATTC & 4598 \\
\hline ompl & CATATGCCTGTAGGGAACCCAGCTGAACC & GGGCGAATTCTTATGCGAATGGAT & 1245 \\
\hline $16 S$ & GGCGGCGTGGATGAGGC & GAGTGTACTCCTCAGGCGGCA & 868 \\
\hline
\end{tabular}

* For constructs cloned into pET30-Ek/Lic vector: forward primers contain 5'-GACGACGACAAGAT-3'; and reverse primers contain 5'-GAG GAGAAGCCCGGT-3'. 
Table II. Real-time PCR primers and probes.

\begin{tabular}{|c|c|c|c|c|}
\hline Gene & Forward primer $\left(5^{\prime}-3^{\prime}\right)$ & Reverse primer $\left(5^{\prime}-3^{\prime}\right)$ & Taqman probe $\left(5^{\prime}-3^{\prime}\right)$ & $\begin{array}{c}\text { Amplicon } \\
\text { size (bp) }\end{array}$ \\
\hline PmplB & GCTACAGAGGATCGGGATTAAGTG & TCCGCTTGCATCTTGTGTTTG & TTCAGATTCTGCATCAAAGAATTTCACGCA & 77 \\
\hline pmp $2 A$ & TTTAGCGAAAACTCCGCAGAA & AATGGGCTGGGAAGCTATAAGG & CGGTGGAGCTATAACCGCGAGCAG & 74 \\
\hline pmp $3 E$ & GGTGGCCAAGGCTTGGT & CGGAATCCTGGCACACCTA & ACGGCTATCCACCAAGCGAATCGC & 61 \\
\hline pmp 4 E & AGGATACATTCCCAACCCCATA & TAGGCGGCTTGCCAAAGA & ATCGTGGAGATCTTGTGGCTAATG & 65 \\
\hline pmp $5 E$ & TCCGTCACTTTGGGAATTAGAAG & TCCGTCACTTTGGGAATTAGAAG & TGCGTATAAACCTAACGCCGTCCGAA & 74 \\
\hline pmp6H & TGCCTCATGCAATATCGAAAAT & GCCGCAAGCAGCAAAGTT & ACTCTGCGGCTATCTCGTTTTCT & 66 \\
\hline pmp $7 G$ & TGCTGCTGAGGTAAGTAGCGTTA & GCTATTCGCTTGATTGGTTACAGA & CGTCGACTGCAGCTACTGCGGC & 72 \\
\hline pmp10G & GCCAACCACTCCTTAATATTTGAAGA & TGGTTTTGCCATCCGTATTTG & ATCGTCTCTACAGCTCAAGGAGCTGCTATCAG & 81 \\
\hline pmp11G & TGCCTTATCCACTGCTACCAAA & GGAGCCGTTGAATCCAGTTG & TTCTTGCAGATGCCGACTCTGTCAACC & 72 \\
\hline pmp13G & TCTTTAATCCTTAAAGATGGTGTCACTCT & CGTAGTCCCTAGGTCCATGACAA & AAATCCTTCACGCAAACAGAGGGCG & 89 \\
\hline pmp14G & GGAGCATTCAGTCCGCAATC & TCTACAATAGAAATATCACTCTCGACGTT & ACAAGCACTGCGGGAGGAACGATTT & 76 \\
\hline pmp15G & СCСТTATCTTTCACCGCATCA & TCGGTGGTGTTTTGGTTTCA & CGGATGTTGCCAATGAGCATGGC & 75 \\
\hline pmp16G & CTCAATGCTAAAGAAGGTTTTGGTATT & GAGAAGACGATCTTTCCTGTATAAGTAGTG & TCTTTTATGACCCTATCGCTAACACAGGAGGATC & 124 \\
\hline pmp17G & GGCACAGGGAAATTTACAAAGC & CTCCCCCAGTAATAGGGTCATAGA & ACGTGCTAAAGACGGCTTCGGAATTTTC & 75 \\
\hline pmp18D & TCCACTGGGATGATCACCAATA & GCATAGAAAGCGTATCGAGAATCAC & TGATAAATTATATGGTTATGTACCAGCTTC & 81 \\
\hline ompl & GCGGCATTCAACCTCGTT & CCTTGAGTGATGCCTACATTGG & TGTTAAAGGATCCTCCATAGCAGCTGATCAG & 85 \\
\hline $16 S$ & AATCTTTCGCAATGGACGAAA & ACAACCCTAGAGCCTTCATCACA & TCTGACGAAGCAACGCCGCG & 66 \\
\hline
\end{tabular}



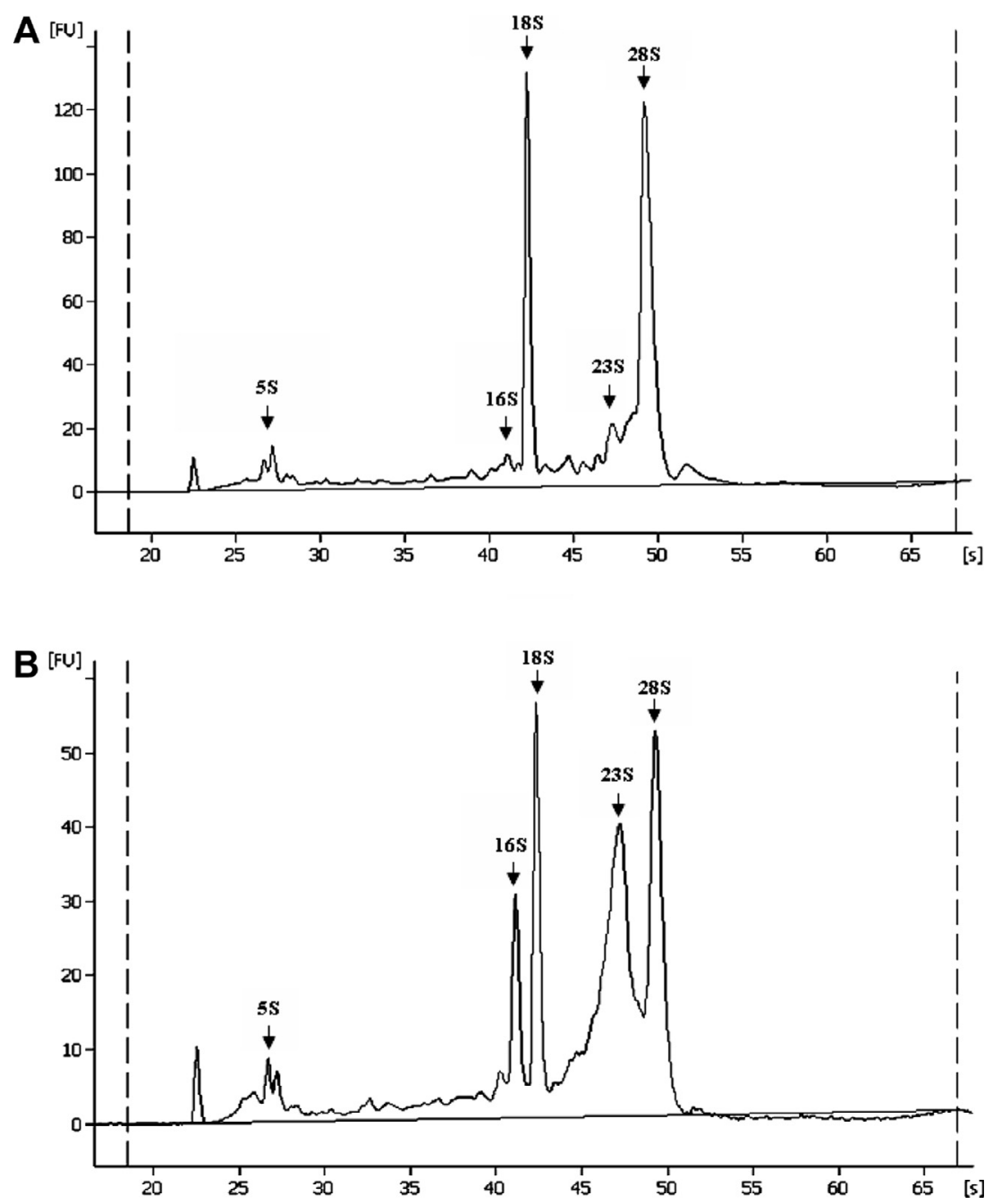

Figure 1. Electropherograms of total RNA isolated from C. abortus-infected McCoy cells at two time points in the chlamydial developmental cycle. The presence of both eukaryotic $(28 \mathrm{~S}, 18 \mathrm{~S}, 5 \mathrm{~S})$ and prokaryotic (chlamydial) (23S, 16S, 5S) RNA is indicated at $24 \mathrm{~h}$ p.i. (A) and $48 \mathrm{~h}$ p.i. (B).

baseline) and peaking at $48 \mathrm{~h}$ p.i. $(p<0.01)$. After $48 \mathrm{~h}$ there was a dramatic fall in $16 \mathrm{~S}$ rRNA transcript levels, which were not significantly different from baseline levels.

The pattern of ompl mRNA expression was similar to that of 16S (Fig. 2B). Levels of trasnscripts were negligible at both 6 and $12 \mathrm{~h}$ p.i. Between 12 and $36 \mathrm{~h}$ p.i. there was a significant increase in the transcript levels $(p<0.05)$. Lev- els again increased to peak at $48 \mathrm{~h}(p<0.01)$ before decreasing to near base-line levels by $60 \mathrm{~h}$.

\subsection{Expression of pmp transcripts}

Prior to determining relative pmp mRNA expression levels, each of the designed pmp primer/probe sets (Tab. I) was tested for 

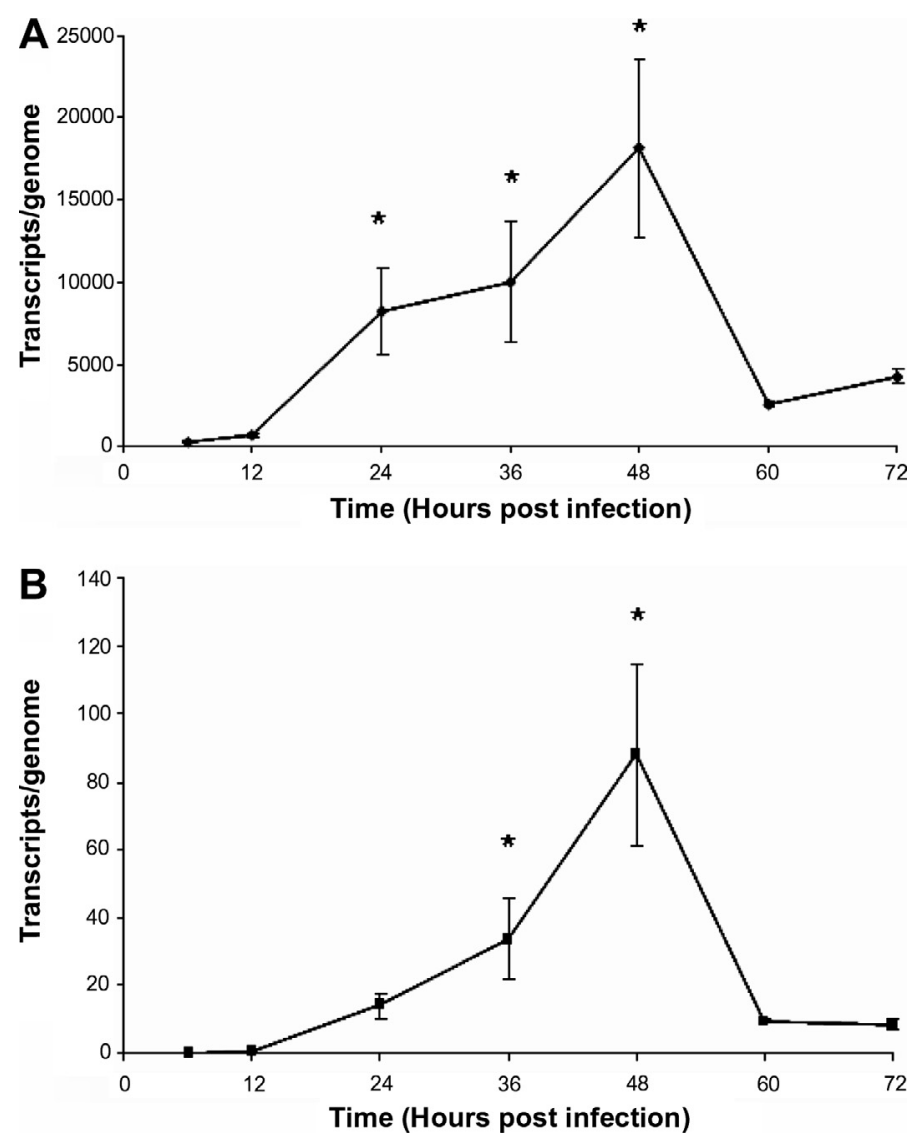

Figure 2. Transcriptional expression of C. abortus $16 S$ rRNA (A) and ompl (B) RNA. Cells were infected with C. abortus (MOI 2). RNA and DNA were harvested between 6 and $72 \mathrm{~h}$ p.i. RNA transcript levels were analyzed by reverse transcription quantitative PCR and normalized against numbers of chlamydial genomes (see Materials and Methods). \pm SEM of three independent experiments (see Materials and Methods). ${ }^{*} p<0.05$ above baseline levels.

cross-reactivity against all 15 cloned plasmid DNA. No cross reactivity was observed with any of the primer/probe sets, each being specific for the relevant plasmid DNA. The results of the real-time PCR assays on the RT reaction mixes for each of the $15 \mathrm{pmp}$ genes, relative to total genome copies at each of the time points in the chlamydial developmental cycle, are shown in Figure 3. No significant $p m p$ gene expression was observed at either the 6 or $12 \mathrm{~h}$ time points. Several pmp genes had a statistically significant increase in expression at $24 \mathrm{~h}, \quad$ specifically $2 A \quad(p<0.05), \quad 10 G$ $(p<0.05), 11 G(p<0.05)$ and $5 E(p<0.01)$. However, only pmp5E exhibited peak expression at this time point, with its expression decreasing throughout the remaining period of the cycle. In contrast, expression of $2 A, 10 G$ and $11 G$ continued to stay elevated albeit at a relatively low level until $48 \mathrm{~h}$, after which expression decreased. For pmp18D expression at $24 \mathrm{~h}$ was much greater than for all other pmps, other than pmp5E, and continued to increase until $48 \mathrm{~h}$ p.i. Maximum expression was observed for all pmps, other than pmp5E, at $48 \mathrm{~h}$ p.i. $(p<0.05)$. As observed for both 

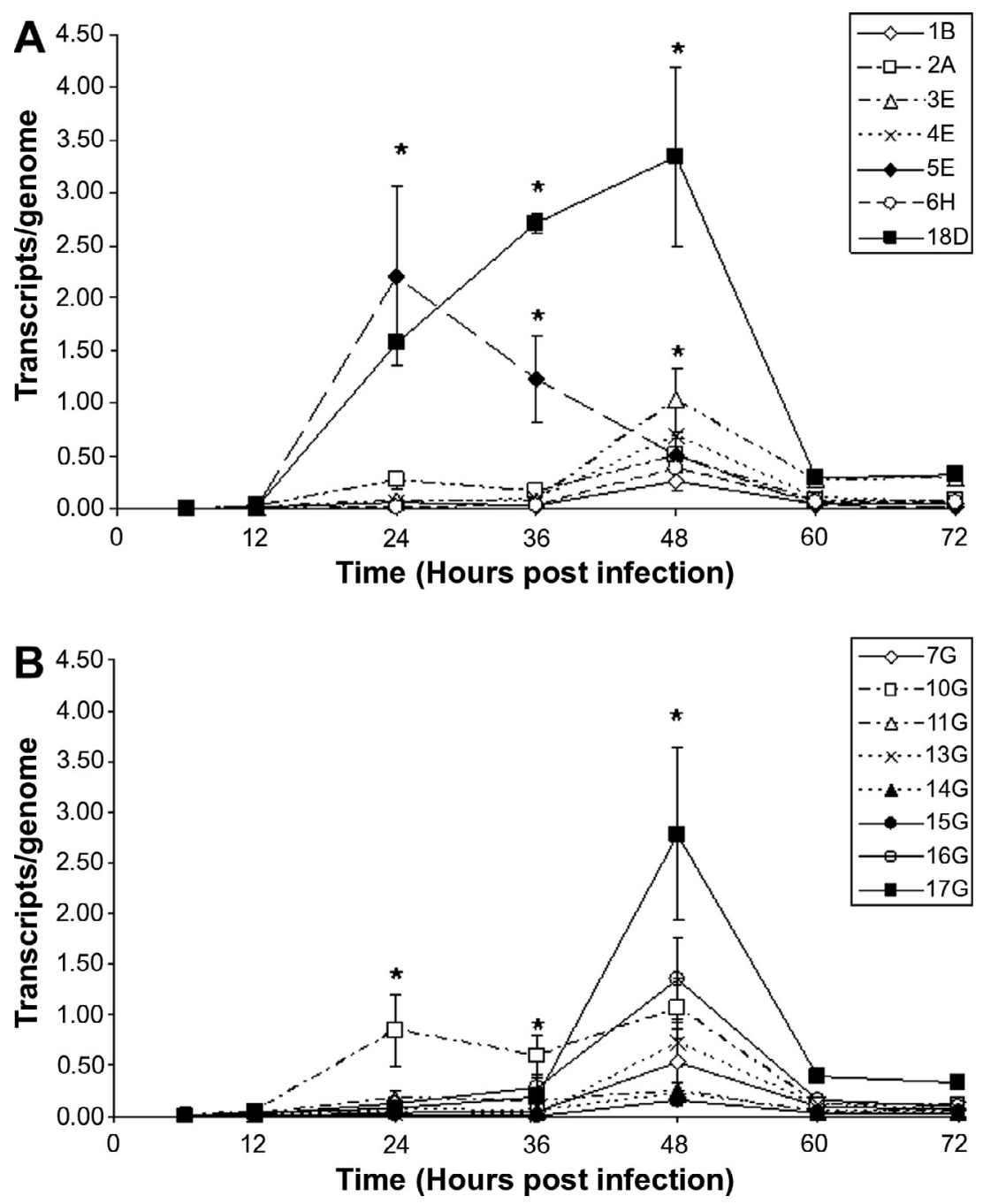

Figure 3. Transcriptional expression of C. abortus pmp mRNA. Cells were infected with C. abortus (MOI 2). RNA and DNA were harvested between 6 and $72 \mathrm{~h}$ p.i. RNA levels were analyzed by reverse transcription quantitative PCR and normalized against numbers of chlamydial genomes (see Materials and Methods). For clarity $p m p$ results have been divided into panels A and B. Panel A depicts non- $p m p G$ family members, while panel B depicts $p m p G$ family members. Results are presented as the mean transcript levels normalized against genome copy number \pm SEM of three independent experiments (see Materials and Methods). ${ }^{*} p<0.05$ above baseline levels.

$16 S$ and ompl, the expression of all the pmps reduced to near base-line levels at the $60 \mathrm{~h}$ time-point and remained low at $72 \mathrm{~h}$. Interestingly, throughout the developmental cycle, transcript levels for all pmps other than pmp5E,
pmp16G, pmp17G and pmp18D, were determined to be below one transcript per genome. Overall, expression of pmps $5 E, 18 D$ and $10 G$ were found to be 40 to 100 -fold higher than the lowest expressing pmps $(6 H, 13 G$ and 
$15 G)$ at $24 \mathrm{~h}$ p.i., while pmps $18 D$ and $17 G$ were 14 to 16-fold higher than the lowest $(11 G, 14 G$ and $15 G)$ at $48 \mathrm{~h}$.

\section{DISCUSSION}

This study has demonstrated the transcriptional expression of $15 \mathrm{pmp}$ genes. Although 18 genes were identified from the sequencing of the $C$. abortus genome [31], one was a duplicate ( $p m p 12 G)$ of $p m p 17 G$, while another two ( $p m p 8 G$ and $p m p 9 G$ ) had unrecoverable frameshifts, and so were not included in this study. Transcripts for all $15 \mathrm{pmp}$ genes were analysed at different stages in the chlamydial developmental cycle: defined as early $(0-12 \mathrm{~h}$ p.i.); mid to late cycle (during the end phase of the growth and multiplication of RB) (12-24 h p.i.); late (terminal differentiation of RB to EB) $(24-48 \mathrm{~h}$ p.i.); and end of cycle (48-72 h p.i.).

Few $p m p$ transcripts were detected in the early stages of the developmental cycle. Indeed the level of transcripts for the majority of the pmp genes was very low (less than 1 copy per genome). This could be due to the asynchronicity of the developmental cycle, although this was kept to a minimum by ensuring a low passage level. It is also highly probable that across the population of $C$. abortus there will be considerable variation in the levels of Pmp expression in individual cells and across the population of cells, which could play an important role in contributing towards antigenic variation. In addition, although McCoy cells are widely used for the growth of $C$. abortus, this organism can infect a wide variety of other cell types in which a different expression profile may have been observed. This will require further investigations, as well as to determine comparative protein expression patterns.

Based upon the premise that 16S rRNA transcripts are constitutively expressed throughout the cycle, other studies have used this against which to normalise their gene of interest [18, 20]. In those studies fixed concentrations of chlamydial RNA were used in the final analyses and it is highly probable that ribosomal RNA does make up a stable component of the chlamydial RNA pool. Whilst this approach illus- trates comparative differences in transcript levels of particular genes of interest within a fixed pool of RNA at a particular time point, it is more difficult to compare transcript levels across time points due to fluctuations in the levels of total RNA and 16S rRNA. The methodology adopted in this study, where both DNA and RNA were isolated from duplicate cultures, allowed the direct determination of transcript numbers relative to the number of organisms present (as determined by genome copy number). The results clearly demonstrate that $16 \mathrm{~S}$ rRNA transcript levels vary throughout the cycle, increasing to around $48 \mathrm{~h}$ and thereafter falling back to low levels reflecting conversion of the RB to $\mathrm{EB}$ and consequential reduction in metabolic activity.

All pmps demonstrated a peak in transcript levels during the mid to late cycle $(24-48 \mathrm{~h}$ p.i.). These results are in agreement with previous studies carried out at both the proteomic [29, 30, 32] and transcriptomic levels [19] for other chlamydial species (C. trachomatis, C. psittaci and C. pneumoniae). The function(s) of the Pmps remains to be elucidated however it is attractive to speculate that the relatively late timing of their mRNA expression is consistent with their presence in either the infectious EB or their requirement in part of the differentiation process, and so potential roles in adhesion, antigenic variation and immune evasion.

Whilst transcript levels of several pmps were significantly up-regulated by $24 \mathrm{~h}$ the level of expression of $p m p 5 E$ at this point was most dramatic. The structure of this particular Pmp is divergent from the other family members in that the protein consists of only a beta-barrel and autochaperone domain and no apparent passenger domain (effector protein). Given the lack of a passenger domain it has been hypothesised that pmp5E represents an orphan gene [31]. However, in the closely related $C$. caviae sequence there is also a $p m p E / F$ gene encoding a truncated Pmp [24]. It is plausible that the truncated Pmp5E may fulfil an as yet undefined role in chlamydial development and could hypothetically function as part of a two-partner secretion system with a yet to be identified partner or could also act as a porin rather than as a classical autotransporter. 
The original characterised Pmp proteins identified in $C$. abortus were those now termed Pmp12/17G (Pomp90A/B), Pmp13G (Pomp91A) and Pmp16G (Pomp91B) [15]. Pmps 12/17G and $16 \mathrm{G}$ were identified as being highly expressed through their immunogenicity with convalescent sheep serum [14] and shown to be highly immunodominant components of a protective chlamydial outer membrane protein preparation [15]. This is consistent with the elevated levels of transcriptional expression observed for pmps $12 / 17 \mathrm{G}$ and $16 \mathrm{G}$, relative to the other pmps (with the exception of pmp18D). Interestingly, Pmp16G was identified in the recently published $C$. abortus genome as a pseudogene by virtue of a frame-shift in a centrally located polyguanine tract [31], while no such frame-shift was observed when originally identified through expression analysis and sequencing [15]. Indeed, Pmp16G has also been reported to be expressed as a full length protein following 2-dimensional gel electrophoresis of whole C. abortus EB preparations [6]. In this current study, while we have demonstrated the expression of pmp16G transcripts we have not examined whether any of them contain a frame-shift mutation that would result in a truncated protein. Phase-variation of protein expression has been observed in the pmp genes of other chlamydial species containing homopolymeric tracts [22] and so could also be a mechanism by which they contribute to antigenic variation.

In comparison with omp 1, the expression of each of the pmps was very low (up to 450 -fold at $48 \mathrm{~h}$ ). However in comparison to the other pmps the expression of pmp $18 D$ was relatively high from mid to late in the cycle. PmpD is the most highly conserved of all the Pmps at both the DNA and amino acid level across the Chlamydiaceae and perhaps this level of conservation combined with the relatively high abundance of transcripts may point to an essential role in the chlamydial developmental cycle. A putative role for PmpD in the adhesion of Chlamydia to host cells has been hypothesised, as antibodies directed against the N-terminal domain have been at least partially successful in inhibiting the in vitro infection of both C. pneumoniae [33] and C. trachomatis [3]. However, the expression of this gene earlier in the cycle would perhaps suggest an additional role for this protein during the late replicative/early differentiation phase.

The results presented in this study represent the most extensive transcriptional characterisation of the C. abortus pmps during the developmental cycle. The data indicates that most of the Pmps are expressed during the later stages of the cycle and thus are likely to play important roles in either the RB or the conversion of $\mathrm{RB}$ to $\mathrm{EB}$ and that the low level of expression may be indicative of a mechanism of antigenic variation. Whilst this is an important step forward in our understanding of the regulation of $\mathrm{pmp}$ gene expression in C. abortus it will be important to follow up these analyses with further studies aimed at investigating protein expression.

Acknowledgements. This work was funded by the Scottish Government Rural and Environment Research and Analysis Directorate (RERAD) and by grant No. BB/E018939/1 from the Biological and Biotechnology Sciences Research Council (BBSRC). We would like to acknowledge the support of the Moredun Proteomics Facility for the sequencing of all plasmid clones used in this study.

\section{REFERENCES}

[1] Aitken I.D., Longbottom D., Chlamydial abortion, in: Aitken I.D. (Ed.), Diseases of sheep, Blackwell Publishing, Oxford, UK, 2007, pp. 105-111.

[2] Azuma Y., Hirakawa H., Yamashita A., Cai Y., Rahman M.A., Suzuki H., et al., Genome sequence of the cat pathogen, Chlamydophila felis, DNA Res. (2006) 13:15-23.

[3] Crane D.D., Carlson J.H., Fischer E.R., Bavoil P., Hsia R.C., Tan C., et al., Chlamydia trachomatis polymorphic membrane protein $\mathrm{D}$ is a speciescommon pan-neutralizing antigen, Proc. Natl. Acad. Sci. USA (2006) 103:1894-1899.

[4] Ehricht R., Slickers P., Goellner S., Hotzel H., Sachse K., Optimized DNA microarray assay allows detection and genotyping of single PCR-amplifiable target copies, Mol. Cell. Probes (2006) 20:60-63.

[5] Entrican G., Immune regulation during pregnancy and host-pathogen interactions in infectious abortion, J. Comp. Pathol. (2002) 126:79-94.

[6] Giannikopoulou P., Bini L., Simitsek P.D., Pallini V., Vretou E., Two-dimensional electrophoretic analysis of the protein family at $90 \mathrm{kDa}$ of 
abortifacient Chlamydia psittaci, Electrophoresis (1997) 18:2104-2108.

[7] Gomes J.P., Nunes A., Bruno W.J., Borrego M.J., Florindo C., Dean D., Polymorphisms in the nine polymorphic membrane proteins of Chlamydia trachomatis across all serovars: evidence for serovar Da recombination and correlation with tissue tropism, J. Bacteriol. (2006) 188:275-286.

[8] Goodall J.C., Yeo G., Huang M.L., Raggiaschi R., Gaston J.S.H., Identification of Chlamydia trachomatis antigens recognized by human $\mathrm{CD} 4(+) \mathrm{T}$ lymphocytes by screening an expression library, Eur. J. Immunol. (2001) 31:1513-1522.

[9] Graham S.P., Jones G.E., MacLean M., Livingstone M., Entrican G., Recombinant ovine interferon gamma inhibits the multiplication of Chlamydia psittaci in ovine cells, J. Comp. Pathol. (1995) 112:185-195.

[10] Henderson I.R., Lam A.C., Polymorphic proteins of Chlamydia spp. - autotransporters beyond the Proteobacteria, Trends Microbiol. (2001) 9: 573-578.

[11] Henderson I.R., Navarro-Garcia F., Desvaux M., Fernandez R.C., Ala'Aldeen D., Type V protein secretion pathway: the autotransporter story, Microbiol. Mol. Biol. Rev. (2004) 68:692-744.

[12] Livingstone M., Entrican G., Wattegedera S., Buxton D., McKendrick I.J., Longbottom D., Antibody responses to recombinant protein fragments of the major outer membrane protein and polymorphic outer membrane protein POMP90 in Chlamydophila abortus-infected pregnant sheep, Clin. Diagn. Lab. Immunol. (2005) 12:770-777.

[13] Livingstone M., Wheelhouse N., Maley S.W., Longbottom D., Molecular detection of Chlamydophila abortus in post-abortion sheep at oestrus and subsequent lambing, Vet. Microbiol. (2009) 135: 134-141.

[14] Longbottom D., Russell M., Jones G.E., Lainson F.A., Herring A.J., Identification of a multigene family coding for the $90 \mathrm{kda}$ proteins of the ovine abortion subtype of Chlamydia psittaci, FEMS Microbiol. Lett. (1996) 142:277-281.

[15] Longbottom D., Russell M., Dunbar S.M., Jones G.E., Herring A.J., Molecular cloning and characterization of the genes coding for the highly immunogenic cluster of 90-kilodalton envelope proteins from the Chlamydia psittaci subtype that causes abortion in sheep, Infect. Immun. (1998) 66:1317-1324.

[16] Longbottom D., Coulter L.J., Animal chlamydioses and zoonotic implications, J. Comp. Pathol. (2003) 128:217-244.
[17] Longbottom D., Livingstone M., Vaccination against chlamydial infections of man and animals, Vet. J. (2006) 171:263-275.

[18] Nicholson T.L., Olinger L., Chong K., Schoolnik G., Stephens R.S., Global stage-specific gene regulation during the developmental cycle of Chlamydia trachomatis, J. Bacteriol. (2003) 185: 3179-3189.

[19] Nicholson T.L., Chiu K., Stephens R.S., Chlamydia trachomatis lacks an adaptive response to changes in carbon source availability, Infect. Immun. (2004) 72:4286-4289.

[20] Nunes A., Gomes J.P., Mead S., Florindo C., Correia H., Borrego M.J., Dean D., Comparative expression profiling of the Chlamydia trachomatis pmp gene family for clinical and reference strains, PLOS ONE (2007) 2:e878.

[21] Nunes A., Nogueira P.J., Borrego M.J., Gomes J.P., Chlamydia trachomatis diversity viewed as a tissue-specific coevolutionary arms race, Genome Biol. (2008) 9:R153.

[22] Pedersen A.S., Christiansen G., Birkelund S., Differential expression of Pmp10 in cell culture infected with Chlamydia pneumoniae CWL029, FEMS Microbiol. Lett. (2001) 203:153-159.

[23] Read T.D., Brunham R.C., Shen C., Gill S.R., Heidelberg J.F., White O., et al., Genome sequences of Chlamydia trachomatis MoPn and Chlamydia pneumoniae AR39, Nucleic Acids Res. (2000) 28:1397-1406.

[24] Read T.D., Myers G.S., Brunham R.C., Nelson W.C., Paulsen I.T., Heidelberg J., et al., Genome sequence of Chlamydophila caviae (Chlamydia psittaci GPIC): examining the role of niche-specific genes in the evolution of the Chlamydiaceae, Nucleic Acids Res. (2003) 31:2134-2147.

[25] Sachse K., Vretou E., Livingstone M., Borel N., Pospischil A., Longbottom D., Recent developments in the laboratory diagnosis of chlamydial infections, Vet. Microbiol. (2009) 135:2-21.

[26] Saren A., Pascolo S., Stevanovic S., Dumrese T., Puolakkainen M., Sarvas M., et al., Identification of Chlamydia pneumoniae-derived mouse CD8 epitopes, Infect. Immun. (2002) 70:3336-3343.

[27] Stephens R.S., Kalman S., Lammel C., Fan J., Marathe R., Aravind L., et al., Genome sequence of an obligate intracellular pathogen of humans: Chlamydia trachomatis, Science (1998) 282:754-759.

[28] Stothard D.R., Toth G.A., Batteiger B.E., Polymorphic membrane protein $\mathrm{H}$ has evolved in parallel with the three disease-causing groups of 
Chlamydia trachomatis, Infect. Immun. (2003) 71:1200-1208.

[29] Tanzer R.J., Hatch T.P., Characterization of outer membrane proteins in Chlamydia trachomatis LGV serovar L2, J. Bacteriol. (2001) 183:2686-2690.

[30] Tanzer R.J., Longbottom D., Hatch T.P., Identification of polymorphic outer membrane proteins of Chlamydia psittaci 6BC, Infect. Immun. (2001) 69: 2428-2434.

[31] Thomson N.R., Yeats C., Bell K., Holden M.T., Bentley S.D., Livingstone M., et al., The Chlamydophila abortus genome sequence reveals an array of variable proteins that contribute to interspecies variation, Genome Res. (2005) 15:629-640.

[32] Vandahl B.B., Pedersen A.S., Gevaert K., Holm A., Vandekerckhove J., Christiansen G., Birkelund S., The expression, processing and localization of polymorphic membrane proteins in Chlamydia pneumoniae strain CWL029, BMC Microbiol. (2002) 2:36.

[33] Wehrl W., Brinkmann V., Jungblut P.R., Meyer T.F., Szczepek A.J., From the inside out - processing of the Chlamydial autotransporter PmpD and its role in bacterial adhesion and activation of human host cells, Mol. Microbiol. (2004) 51:319-334. 